\title{
Dual-wavelength bidirectional pumped high-power Raman fiber laser
}

\author{
Zehui Wang, Qirong Xiao, Yusheng Huang, Jiading Tian, Dan Li, Ping Yan, and Mali Gong \\ State Key Laboratory of Precision Measurement Technology and Instruments \& Key Laboratory of Photonics Control Technology of \\ the Ministry of Education, Tsinghua University, Beijing 100084, China \\ (Received 21 September 2018; revised 12 November 2018; accepted 21 November 2018)
}

\begin{abstract}
In this paper, we reported both the experimental demonstration and theoretical analysis of a Raman fiber laser based on a master oscillator-power amplifier configuration. The Raman fiber laser adopted the dual-wavelength bidirectional pumping configuration, utilizing $976 \mathrm{~nm}$ laser diodes and $1018 \mathrm{~nm}$ fiber lasers as the pump sources. A 60-m-long $25 / 400 \mu \mathrm{m}$ ytterbium-doped fiber was used to convert the power from 1070 to $1124 \mathrm{~nm}$, realizing a maximum power output of $3.7 \mathrm{~kW}$ with a $3 \mathrm{~dB}$ spectral width of $6.8 \mathrm{~nm}$. Moreover, we developed a multi-frequency model taking into consideration the Raman gain spectrum and amplified spontaneous emission. The calculated spectral broadening of both the forward and backward laser was in good agreement with the experimental results. Finally, a $1.5 \mathrm{~kW}, 1183 \mathrm{~nm}$ second-order Raman fiber laser was further experimentally demonstrated by the addition of a 70-m-long germaniumdoped passive fiber.
\end{abstract}

Keywords: fiber laser; fiber optics amplifiers and oscillators; Raman laser

\section{Introduction}

Lasers with wavelengths ranging from 1100 to $1300 \mathrm{~nm}$ are promising for applications such as remote sensing ${ }^{[1]}$, spectral beam combining ${ }^{[2]}$, and $\mathrm{Tm}_{-}^{-[3,4]}$ and $\mathrm{Ho}^{[4-7]}$-doped fiber laser pumping. In general, ytterbium-doped fiber lasers (YDFLs) are considered the most promising candidates for achieving high output powers based on the master oscillator power amplifier (MOPA) configuration ${ }^{[8]}$. However, traditional YDFLs can only efficiently operate within the band of 1000-1100 $\mathrm{nm}$. The small emission cross-section of ytterbium and the amplified spontaneous emission (ASE) may deter YDFLs from realizing high output powers when the lasing wavelength exceeds $1100 \mathrm{~nm}^{[9]}$. Nevertheless, it has become a trend to realize lasers of a wide range of wavelengths by taking advantage of the stimulated Raman scattering (SRS) effect with appropriate and available pump sources. As the fiber power coupler replaces the wavelength division multiplexer (WDM), the Raman fiber laser has made significant progress in recent years.

In 2014, Zhang et al. proposed a 732-W integrated Yb-Raman fiber amplifier with dual-wavelength seeds of 1080 and $1120 \mathrm{~nm}$. The length of the 20/400 $\mu \mathrm{m}$ Yb-doped

Correspondence to: Q. Xiao, Department of Precision Instrument, Tsinghua University, Beijing 100084, China.

Email: xiaoqirong08@gmail.com fiber (YDF) in their amplifier was $45 \mathrm{~m}^{[10]}$. Subsequently, in 2015, a $1.5 \mathrm{~kW}$ Raman fiber laser was realized by using the same experimental configuration ${ }^{[11]}$. In 2014, Zhang et al. injected a seed laser at $1120 \mathrm{~nm}$ into a $1080 \mathrm{~nm} \mathrm{Yb}$ doped fiber MOPA consisting of a $12 \mathrm{~m}$ YDF and $70 \mathrm{~m}$ germanium-doped fiber (GDF) with the same parameter of 20/400 $\mu \mathrm{m}$. As a result, a $1.28 \mathrm{~kW} 1120 \mathrm{~nm}$ Raman laser was realized ${ }^{[12]}$. In 2015, Ma et al. designed linearly polarized output $\mathrm{Yb}$-Raman cascaded oscillators consisting of a $1120 \mathrm{~nm}$ Raman Stokes cavity and $1080 \mathrm{~nm}$ laser cavity. They obtained a $1181 \mathrm{~W}, 1120 \mathrm{~nm}$ laser with an opticalto-optical efficiency of $74.3 \%$ using a 21-m-long 20/400$\mu \mathrm{m} \mathrm{YDF}{ }^{[13]}$. It should be noted that all the approaches mentioned above adopt the forward-pumped configuration, in which the output laser is in the same direction as the pump laser. Both theoretical ${ }^{[14]}$ and experimental results ${ }^{[15]}$ prove that the backward pumping Raman threshold should be larger than that of the forward pumping. However, the relatively small gap between the first- and second-order Raman threshold limits the power scaling of the first-order Raman wave. Thus, backward pumping or bidirectional pumping is an effective and competitive method to address the problem, which presents yet another challenge for the backward fiber coupler.

In 2016, our group realized a bidirectional pumped Raman fiber laser, which consisted of a single-wavelength seed and 
a one-stage bidirectional pumped amplifier. A 30-m-long $20 / 400 \mu \mathrm{m}$ YDF was adopted in the amplifier. Under a pump power of $5487 \mathrm{~W}$, a $3889 \mathrm{~W}$ output power of the first-order Raman amplification was achieved with an optical-to-optical efficiency of $70.9 \%{ }^{[15]}$.

In the aforementioned examples, the adopted YDF had a relatively small core size of $20 \mu \mathrm{m}$. This is because smaller core sizes, as well as longer-gain fibers, can induce higher Raman gains. However, the accompanying selfphase modulation and four-wave multiplexing also induce spectral broadening because of the high laser density in the small-core-size fiber. In some cases, high-power and narrow bandwidth lasers are required. For example, the spectrum beam combining is an effective method to break through the limitation of output power in single mode fiber. And the basic elements of spectrum beam combining are highpower and narrow bandwidth lasers with different operating wavelengths $^{[2]}$. The spectral broadening could be suppressed by using a larger-core-size fiber. In 2018, Glick et al. reported a $1.2 \mathrm{~kW}$ cladding pumped Raman laser by using a 80-m-long specialty multi-layer fiber $(25 / 45 / 250 \mu \mathrm{m})^{[16]}$. Chen et al. proposed a $987 \mathrm{~W}$ Raman laser with a large GRIN fiber ${ }^{[17]}$.

In theory, Ying et al. analyzed the Raman noise-enhanced SRS in a continuous-wave fiber amplifier with a singlefrequency model ${ }^{[14]}$. Zhang et al. ${ }^{[10]}$, Xiao et al. ${ }^{[15]}$, Chen et $a{ }^{\left[{ }^{[18]}\right.}$ and Rini et al. ${ }^{[19]}$ adopted the single-frequency model to calculate the output power of their Raman oscillator or amplifier. However, in the single-frequency model, Raman gain coefficient is simplified to a number irrespective of wavelength. Actually, conventional silica fibers have a wide Raman gain spectrum. Therefore, it is not accurate for power calculation via this model. Besides, the spectrum evolution along the fiber cannot be calculated either.

In this paper, we presented both the experimental realization and theoretical analysis of a $3.7 \mathrm{~kW}, 1123 \mathrm{~nm}$ Raman fiber laser system based on the dual-wavelength bidirectional pumping configuration. A total forward pump power of $4200 \mathrm{~W}$ and a backward pump power of $2510 \mathrm{~W}$ were injected into the amplifier of the system. The forward pumping used long-wavelength fiber laser sources at $1018 \mathrm{~nm}$ for reducing the thermal effect caused by quantum defects. Due to the slow absorption of the $1018 \mathrm{~nm}$ fiber laser, $976 \mathrm{~nm}$ laser diodes (LDs) were used as the backward pump sources to reduce the unabsorbed pump light and provide an enhanced absorption. In the core of YDF, the $1070 \mathrm{~nm}$ signal laser was amplified via $\mathrm{Yb}$ gain and then it was converted to $1123 \mathrm{~nm}$ laser via Raman gain. The system incorporated a 60-m-long 25/400 $\mu \mathrm{m}$ YDF, realizing a $3 \mathrm{~dB}$ spectral width of $6.8 \mathrm{~nm}$. Further, the backward Raman laser had a power of $65 \mathrm{~W}$ with a $3 \mathrm{~dB}$ spectral width of $5.2 \mathrm{~nm}$. Moreover, we developed a multi-frequency model of the Raman fiber laser taking into consideration the Raman gain spectrum and ASE. Using this model, we can simulate the spectrum and power evolution along the fiber. The numerical simulation results were in good agreement with the experimental results. Then we analyzed the new peak at $1095 \mathrm{~nm}$ and we found that it was generated from ASE initially and was strongly amplified along the fiber because of much larger gain coefficient. The new peak only appeared when the gain fiber length was larger than $50 \mathrm{~m}$. In addition, we spliced another GDF after the gain fiber to acquire a high Raman gain for realizing a second-order Raman laser. When the length of the GDF reached $70 \mathrm{~m}$, a $1.5 \mathrm{~kW}, 1187 \mathrm{~nm}$ second-order Raman laser was obtained.

\section{Experimental setup}

The Raman fiber laser employing the MOPA configuration consists of a $1070 \mathrm{~nm}$ laser seed and an amplifier of the dualwavelength bidirectional pumping configuration, as shown in Figure 1. The seed comprising a pair of fiber Bragg gratings (FBGs) is pumped by an LD through a $(2+1) \times 1$ coupler. The maximum output power of the seed is $107.4 \mathrm{~W}$ and the $3 \mathrm{~dB}$ linewidth is $0.9 \mathrm{~nm}$. In the amplifier, a 60-m-long Yb-doped double-cladding fiber $(25 / 400 \mu \mathrm{m})$ is adopted as the gain medium. To reduce the thermal effect caused by quantum defects, $1018 \mathrm{~nm}$ YDFLs $^{[20,21]}$ are used as the forward pump source. The cladding absorption coefficient of YDF at $976 \mathrm{~nm}$ is $1.8 \mathrm{~dB} / \mathrm{m}$. In the experiment, the pump powers of six $1018 \mathrm{~nm}$ YDFLs are coupled into the gain fiber with a $(6+1) \times 1$ coupler. The pump coupler efficiency can reach $99 \%$ with a total forward pump power of $4296 \mathrm{~W}$. To provide an enhanced absorption and reduce the unabsorbed pump light, six $976 \mathrm{~nm}$ LDs provide backward pump power through another $(6+1) \times 1$ coupler. The pump power of each LD is approximately $400 \mathrm{~W}$ and the total backward pump power is $2510 \mathrm{~W}$. All the couplers mentioned above are homemade with an excellent coupling efficiency and very small loss coefficient ${ }^{[22,23]}$. A cladding light stripper (CLS) is spliced with the backward coupler to leak the residual pump laser. At the end of the experimental system, a specially made end cap, whose coating is designed to antireflect the Raman laser, is spliced after the CLS and cooled by water. In addition to the power meter, optical spectrum analyzers are used both before the signal input port of the seed coupler and after the end cap to monitor the real-time forward and backward output spectra, respectively.

\section{Experimental results}

Figure 2 shows the output power in the amplifier varying with the input pump power. In part I of Figure 2, the forward pump power increases to a maximum of $4200 \mathrm{~W}$ while the backward pump power remains disabled. Then, in part II, the backward pump power increases from zero to a maximum of $2510 \mathrm{~W}$ while the forward pump power reaches $4200 \mathrm{~W}$. 


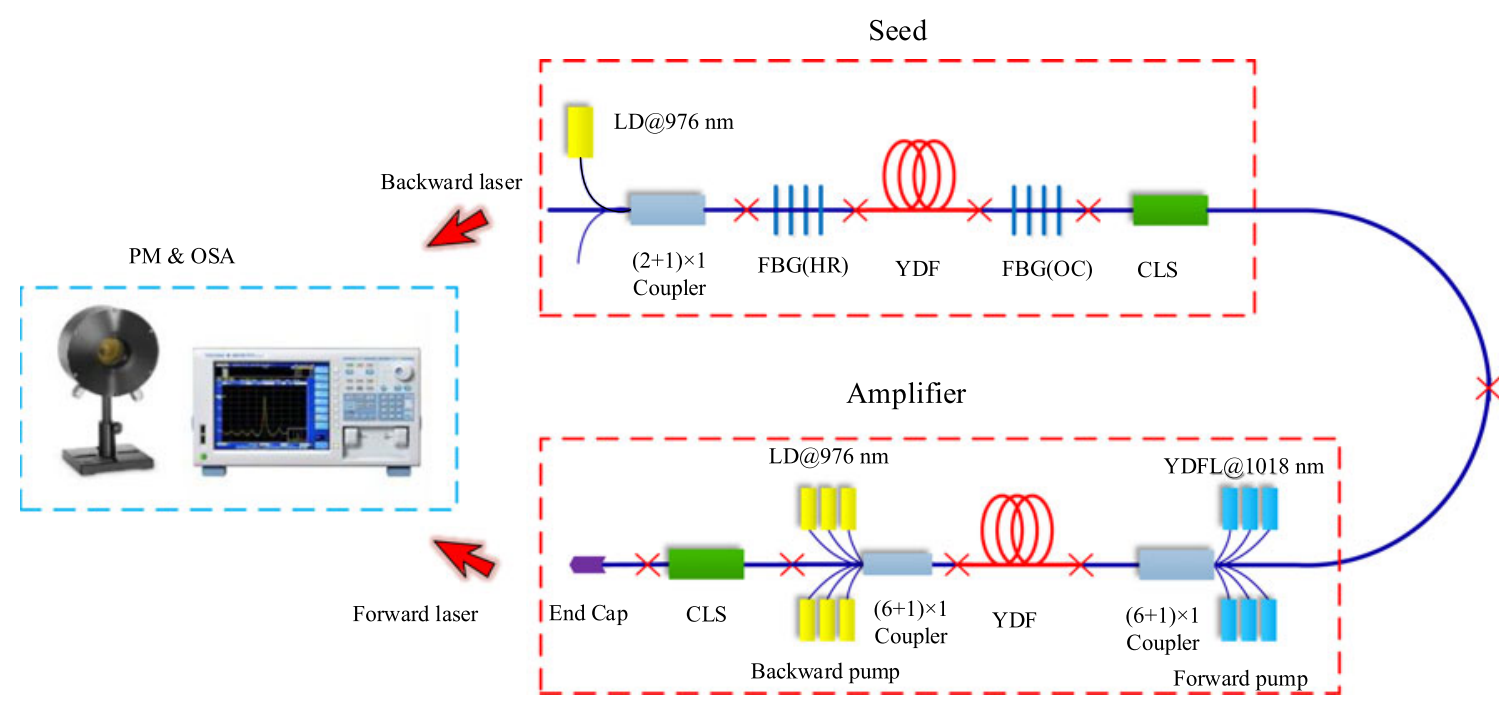

Figure 1. Experimental setup of the Raman laser. PM: power meter, OSA: optical spectrum analyzer, CLS: cladding light stripper, YDFL:Yb-doped fiber laser.

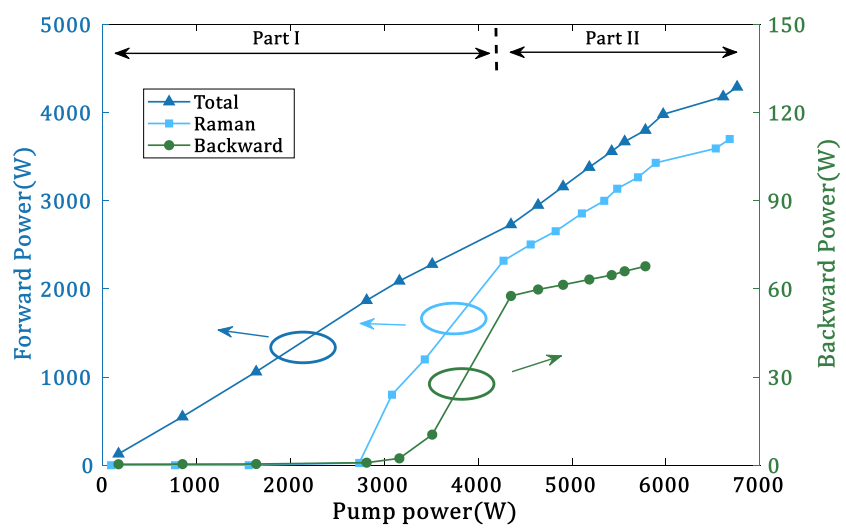

Figure 2. Forward and backward output power as a function of pump power (only forward pumping in part I and both forward and backward pumping in part II).

The figure clearly indicates that the total output power (' $\boldsymbol{\Delta}$ ' in Figure 2) increases almost linearly with the input pump power, and the maximum output power is $4290 \mathrm{~W}$. The output spectrum is depicted as a blue line in Figure 3(a), and no second-order Raman laser is found. Because of the small absorption cross-section at $1018 \mathrm{~nm}$, the slow absorption of the forward pump laser along the fiber length causes some residual pump power. Therefore, we spliced a homemade CLS after the gain fiber. The CLS can leak almost all high numerical aperture (NA) cladding light, but not function well for low NA cladding light. In the experiment, the forward pump source is $1018 \mathrm{~nm}$ fiber laser of high brightness and low NA. Hence, after the CLS, there is still $200 \mathrm{~W}$ residual low NA cladding pump power. It is interesting to note that in addition to the $1070 \mathrm{~nm}$ signal laser, a new peak appears at a wavelength of about $1095 \mathrm{~nm}$, which will be discussed in detail later. The power of the Raman
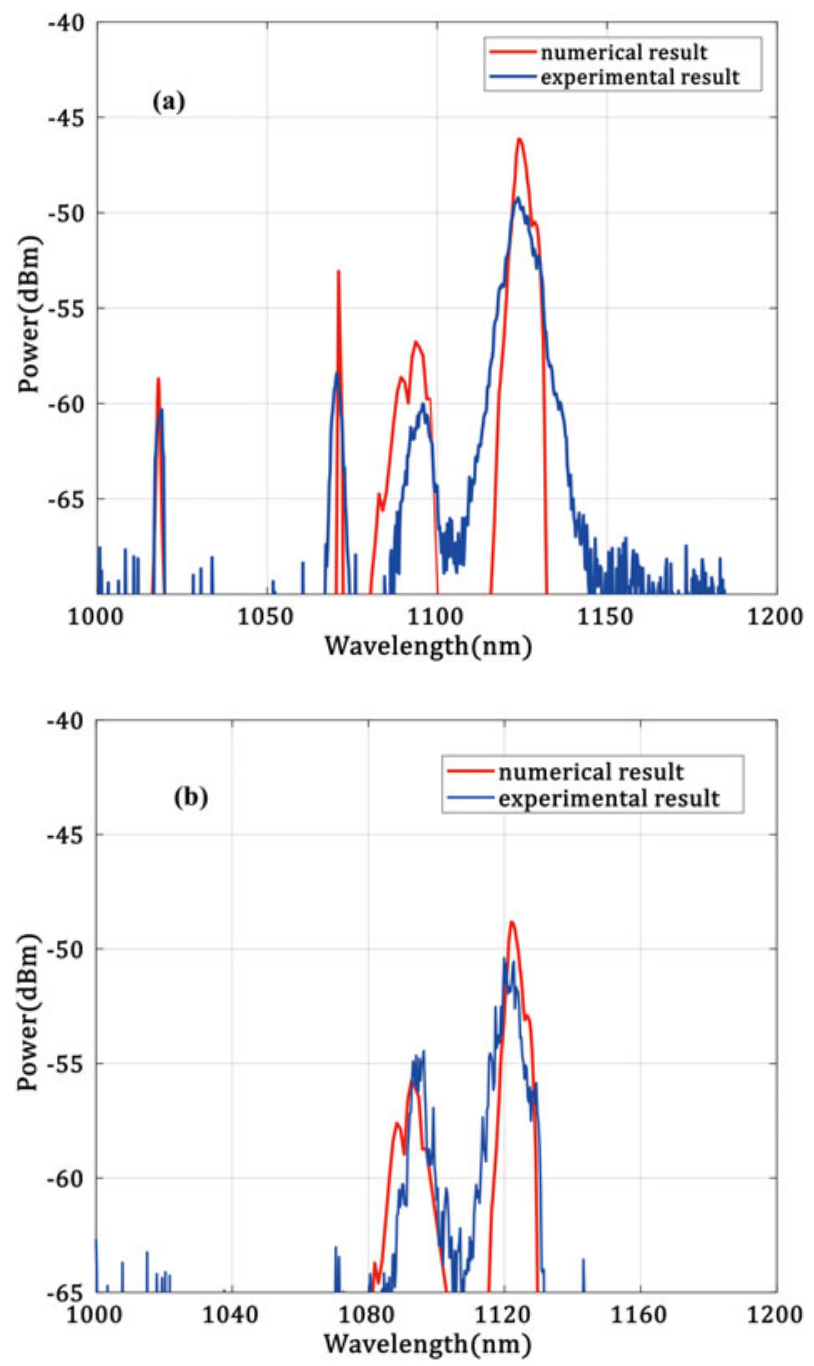

Figure 3. (a) Forward spectrum and (b) backward spectrum. The red and blue lines represent the numerical simulation and experimental results, respectively. 


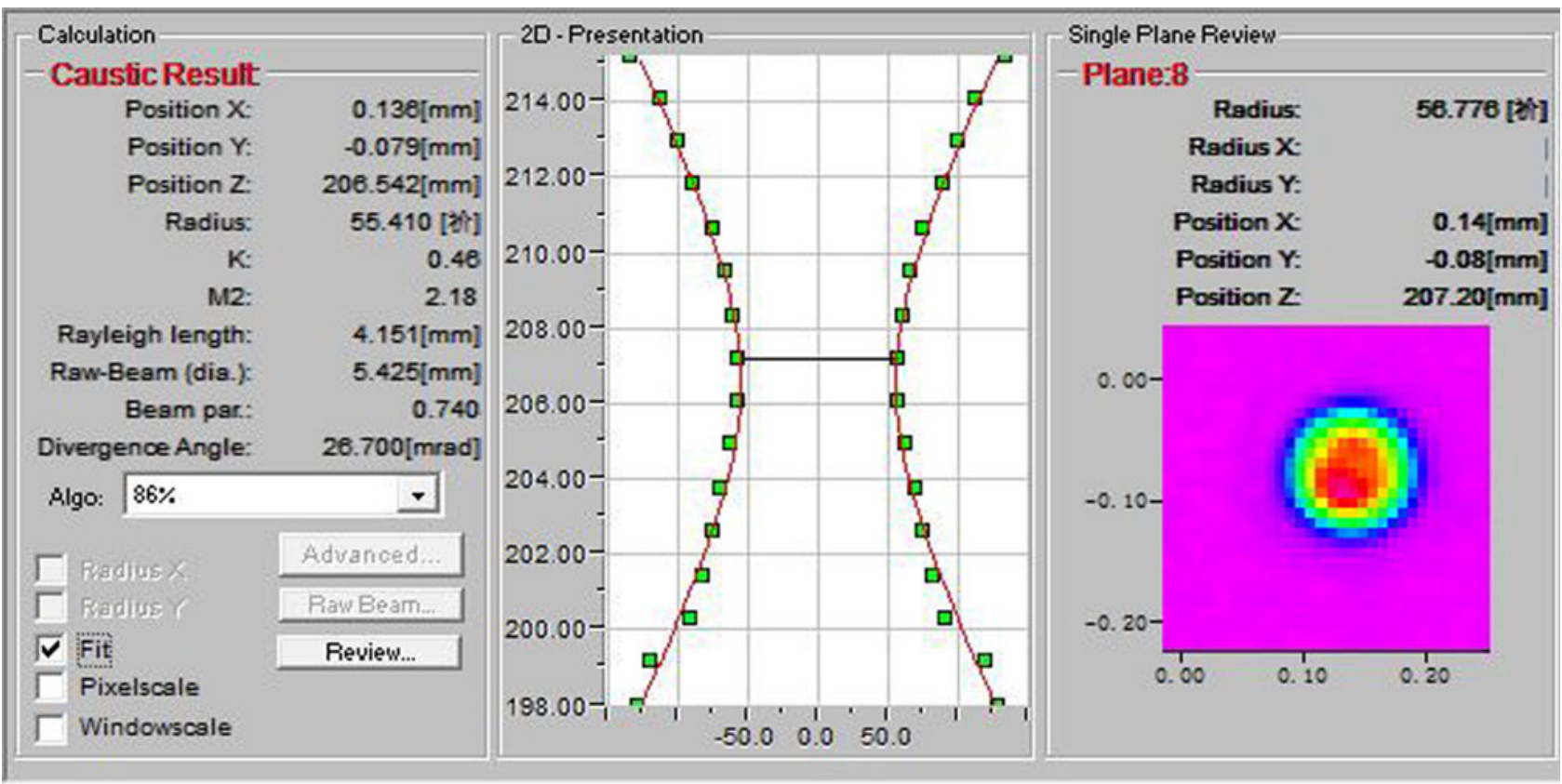

Figure 4. $M^{2}$ factor of output laser.

laser (' $\square$ ' in Figure 2) is approximately 3700 W, which was calculated by spectral integration. The forward first-order Raman threshold is about $3000 \mathrm{~W}$. The center wavelength of the Raman laser is $1123.8 \mathrm{~nm}$ and the $3 \mathrm{~dB}$ width is $6.8 \mathrm{~nm}$, which is much narrower than that in Ref. [15]. We ascribed the narrow output linewidth to the adoption of the $25 / 400 \mu \mathrm{m}$ instead of $20 / 400 \mu \mathrm{m}$ gain fiber, thus leading to a lower power density in the fiber core. We also monitored the backward output power, indicated by the green line (' $O$ ') in Figure 2, and its spectrum is presented in Figure 3(b). Only a Raman laser and new peak near the $1095 \mathrm{~nm}$ laser can be found in the backward spectrum. The center wavelength and $3 \mathrm{~dB}$ spectral width of the backward Raman laser are 1121.9 and $5.2 \mathrm{~nm}$, respectively.

In Figure 2, the curve of the backward power versus pump power can be divided into three sections. The first one is when the forward pump power is below $3000 \mathrm{~W}$, in which the Raman threshold has not been reached and only Raman noise less than $3 \mathrm{~W}$ can be detected. In the second section, the forward pump power rises above the Raman threshold, causing the backward Raman laser power to rapidly amplify to about $65 \mathrm{~W}$. The backward power is much lower than the forward power because the $1070 \mathrm{~nm}$ laser barely propagates in the backward direction. In the third part, when the forward pump power stops rising while the backward pump power starts rising, the power of the backward Raman laser grows slowly. Through the backward power curve and forward Raman power curve, we can easily confirm that the first-order Raman threshold is about $3000 \mathrm{~W}$ in the experiment.

The $M^{2}$ factor of the output laser was measured by a PRIMES High-Power-LaserQualityMonitor. The $M^{2}$ factor of $1070 \mathrm{~nm}$ seed is 1.39 . When the pump power was $3020 \mathrm{~W}$, the $M^{2}$ factor deteriorated to 2.18, as shown in Figure 4. We think that it is a result of thermal effect and the mismatch of the core diameter in the oscillator and amplifier.

\section{Numerical simulation}

We want to figure out the spectral evolution in the fiber amplifier and the existing single-frequency model does not work anymore. Thus, we build the multi-frequency model based on steady-state rate equations to solve the problem. The spectral broadening caused by the Raman gain, ASE, seed and pump light linewidth in the evolutions can be calculated via the multi-frequency model. The model includes the following equations:

$$
\begin{aligned}
\frac{\partial P_{p}^{ \pm}\left(z, \lambda_{p}^{ \pm}\right)}{\partial z}= & \Gamma_{p}\left\{\left[\sigma_{a, p}\left(\lambda_{p}\right)+\sigma_{e, p}\left(\lambda_{p}\right)\right] N_{2}(z)\right. \\
& \left.-\sigma_{a, p}\left(\lambda_{p}\right) N\right\} P_{p}^{ \pm}\left(z, \lambda_{p}\right)-\alpha_{p} P_{p}^{ \pm}\left(z, \lambda_{p}\right)
\end{aligned}
$$

$$
\begin{aligned}
\frac{\partial P_{s}\left(z, \lambda_{s}\right)}{\partial z}= & \Gamma_{s}\left\{\left[\sigma_{a, s}\left(\lambda_{s}\right)+\sigma_{e, s}\left(\lambda_{s}\right)\right] N_{2}(z)\right. \\
& \left.-\sigma_{a, s}\left(\lambda_{s}\right) N\right\} P_{s}\left(z, \lambda_{s}\right)-\alpha_{s} P_{s}\left(z, \lambda_{s}\right) \\
& -\int_{r 1} \frac{\lambda}{\lambda_{s}} \frac{g_{r}\left(\lambda-\lambda_{s}\right)}{A_{\mathrm{eff}}}\left[P_{r 1}^{+}(z, \lambda)+P_{r}^{-}(z, \lambda)\right. \\
& \left.+4 P_{\text {spon } 1}\right] P_{s}\left(z, \lambda_{s}\right) \mathrm{d} \lambda,
\end{aligned}
$$

$$
\begin{aligned}
\frac{\partial P_{r 1}^{ \pm}\left(z, \lambda_{r 1}\right)}{\partial z}= & \pm \Gamma_{r 1}\left\{\left[\sigma_{a, r 1}\left(\lambda_{r 1}\right)+\sigma_{e, r 1}\left(\lambda_{r 1}\right)\right] N_{2}(z)\right. \\
& \left.-\sigma_{a, r 1}\left(\lambda_{r 1}\right) N\right\} P_{r 1}^{ \pm}\left(z, \lambda_{r 1}\right)
\end{aligned}
$$




$$
\begin{aligned}
& \mp \alpha_{r 1} P_{r 1}^{ \pm}\left(z, \lambda_{r 1}\right) \pm \int_{s} \frac{g_{r}\left(\lambda_{r 1}-\lambda_{s}\right)}{A_{\mathrm{eff}}} \\
& \times\left[P_{r 1}^{ \pm}\left(z, \lambda_{r 1}\right)+2 P_{\mathrm{spon}}\right] P_{s}(z, \lambda) \mathrm{d} \lambda \\
& \mp \int_{r 2} \frac{\lambda}{\lambda_{r 1}} \frac{g_{r}\left(\lambda-\lambda_{r 1}\right)}{A_{\mathrm{eff}}}\left[P_{r 2}^{+}(z, \lambda)\right. \\
&\left.+P_{r}^{-}(z, \lambda)+4 P_{\mathrm{spon} 1}\right] P_{r 1}^{ \pm}\left(z, \lambda_{r 1}\right) \mathrm{d} \lambda, \quad(3) \\
& \frac{\partial P_{r 2}^{ \pm}\left(z, \lambda_{r 2}\right)}{\partial z}= \pm \Gamma_{r 2}\left\{\left[\sigma_{a, r 2}\left(\lambda_{r 2}\right)+\sigma_{e, r 2}\left(\lambda_{r 2}\right)\right] N_{2}(z)\right. \\
&\left.-\sigma_{a, r 2}\left(\lambda_{r 2}\right) N\right\} P_{r 2}^{ \pm}\left(z, \lambda_{r 2}\right) \mp \alpha_{r 2} P_{r 2}^{ \pm}\left(z, \lambda_{r 2}\right) \\
& \pm \int_{r 1} \frac{g_{r}\left(\lambda_{r 2}-\lambda_{s}\right)}{A_{\mathrm{eff}}}\left[P_{r 2}^{ \pm}\left(z, \lambda_{r 2}\right)+2 P_{\mathrm{spon}}\right] \\
& \times\left[P_{r 1}^{+}(z, \lambda)+P_{r 1}^{-}(z, \lambda)\right] \mathrm{d} \lambda, \\
& \frac{\partial P_{\mathrm{ase}}^{ \pm}(z, \lambda)}{\partial z \partial \lambda}= \pm \Gamma_{\mathrm{ase}} \sigma_{e, s}\left(\lambda_{s}\right) N_{2}(z) \frac{h c^{2}}{\lambda^{3}} \mp \alpha_{\mathrm{ase}} P_{\mathrm{ase}}^{ \pm}(z, \lambda), \\
& \frac{N_{2}(z)}{N}=\frac{\sum_{i=p^{ \pm}, p^{-}, s, r_{1}^{ \pm}, r_{2}^{ \pm}, \text {ase }} \int_{i} P_{i}(z, \lambda) \sigma_{a, i} \Gamma_{i} \mathrm{~d} \lambda}{\sum_{i=p^{ \pm}, p^{-,}, s, r_{1}^{ \pm}, r_{2}^{ \pm}, \text {ase }} \int_{i} P_{i}(z, \lambda)\left(\sigma_{a, i}+\sigma_{e, i}\right) \Gamma_{i} \mathrm{~d} \lambda+\frac{h c A_{c}}{\tau}}
\end{aligned}
$$

In the above equations, the subscripts $p, s, r 1$ and $r 2$ represent the pump laser, signal laser, first-order Raman wave and second-order Raman wave, respectively. The superscripts ' + ' and ' - ' represent the forward-propagating waves and backward-propagating waves. $\Gamma$ is the filling factor, $\alpha$ is the background loss coefficient, $A_{\text {eff }}$ is the effective mode field area of the core, $N$ is the population density of doped ions, $N_{2}$ is the upper lasing level population density, $\sigma_{a}$ and $\sigma_{e}$ are functions of the wavelength representing the absorption and emission cross-sections, respectively, $h$ is Planck's constant, $c$ is the speed of light in vacuum and $\tau$ is the spontaneous lifetime of doped ions. The subscripts under the integral sign indicate that the integral domain is the wavelength of the corresponding wave. The adopted values of the parameters mentioned are listed in Table 1. The Raman gain is a function of the wavelength shift between the Raman wave and its corresponding Raman pump wave. Figure 5 depicts the Raman gain spectrum in a $25 / 400 \mu \mathrm{m}$ gain fiber with a Raman pump wavelength of about $1070 \mathrm{~nm}^{[24,25]}$.

Table 1. Parameters for the numerical calculations.

\begin{tabular}{ll}
\hline Parameter & Value \\
\hline$\sigma_{a}$ & Ref. [26] \\
$\sigma_{e}$ & Ref. [26] \\
$\Gamma_{S}$ & $0.8^{[27]}$ \\
$\Gamma_{p}$ & $0.05(20 / 400), 0.0625(25 / 400)^{[27]}$ \\
$\Gamma_{r 1}, \Gamma_{r 2}, \Gamma_{\text {ase }}$ & 0.8 \\
$c$ & $2.9979 \times 10^{8} \mathrm{~m} / \mathrm{s}$ \\
$h$ & $6.626 \times 10^{-34} \mathrm{~J} \cdot \mathrm{s}$ \\
$\tau$ & $0.82 \mathrm{~ms}$ \\
\hline
\end{tabular}

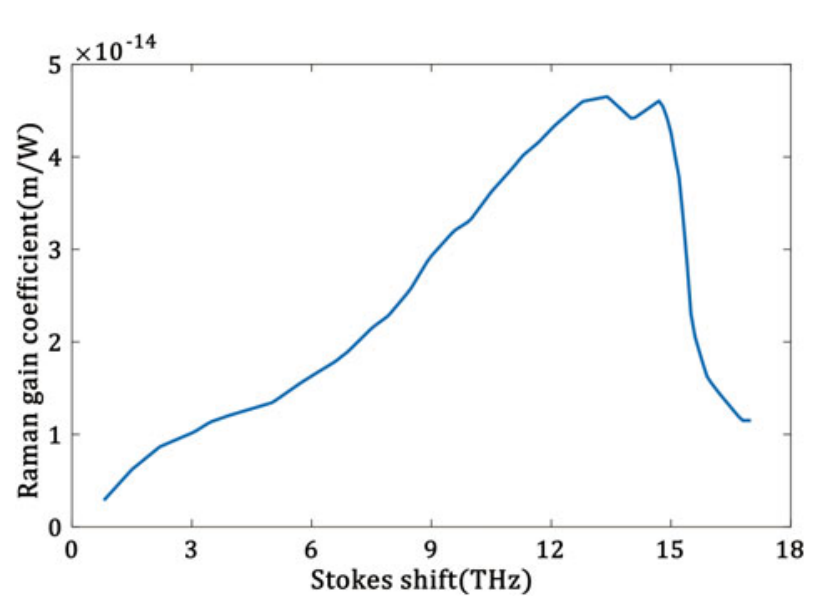

Figure 5. Raman gain spectrum in a $25 / 400 \mu \mathrm{m}$ gain fiber.

\section{Simulation results and discussion}

\subsection{The simulation of the $3.7 \mathrm{~kW}$ bidirectional Raman fiber laser}

Figure 6 illustrates the power distribution along the fiber calculated from the multi-frequency model. The $1070 \mathrm{~nm}$ laser power decreased while the first-order Raman laser power rose rapidly from a fiber length of 45 to $60 \mathrm{~m}$. At the end of the gain fiber, the Raman laser power was calculated to $3850 \mathrm{~W}$ and the backward Raman laser power was calculated to $69 \mathrm{~W}$. The numerical results of the forward and backward spectra were represented by a red line in Figures 3(a) and 3(b), respectively, which agreed with the experiment well.

We also used the model to simulate the spectrum evolution along the fiber, as shown in Figure 7. We can see that the $\mathrm{Yb}$ fiber Raman gain spectrum possessed two peaks where the shift frequencies were 13 and $14.7 \mathrm{THz}$, as shown in Figure $5^{[28-30]}$. Therefore, in the spectrum shown in Figure 7 , next to the main peak of the Raman laser at $1123 \mathrm{~nm}$, there was another peak at $1129 \mathrm{~nm}$. The simulated results of output spectrum for forward and backward direction were shown as red lines in Figures 3(a) and 3(b), respectively. As can be seen clearly, the shapes of the simulated and experimental output spectra were similar. The $3 \mathrm{~dB}$ spectral width was calculated to $5.3 \mathrm{~nm}$, which was little narrower than that of the experimental result $(6.8 \mathrm{~nm})$. We attributed it to omitting other nonlinear effects under the high-intensity light field, which will be left for future research.

\subsection{The comparison of $976 \mathrm{~nm}$ and $1018 \mathrm{~nm}$ pump sources}

In the experiment, we adopted dual-wavelength pump configuration, consisting of $1018 \mathrm{~nm}$ YDFLs and $976 \mathrm{~nm}$ LDs as the forward and backward pump sources, respectively. It should be noted that due to the small quantum defect and 

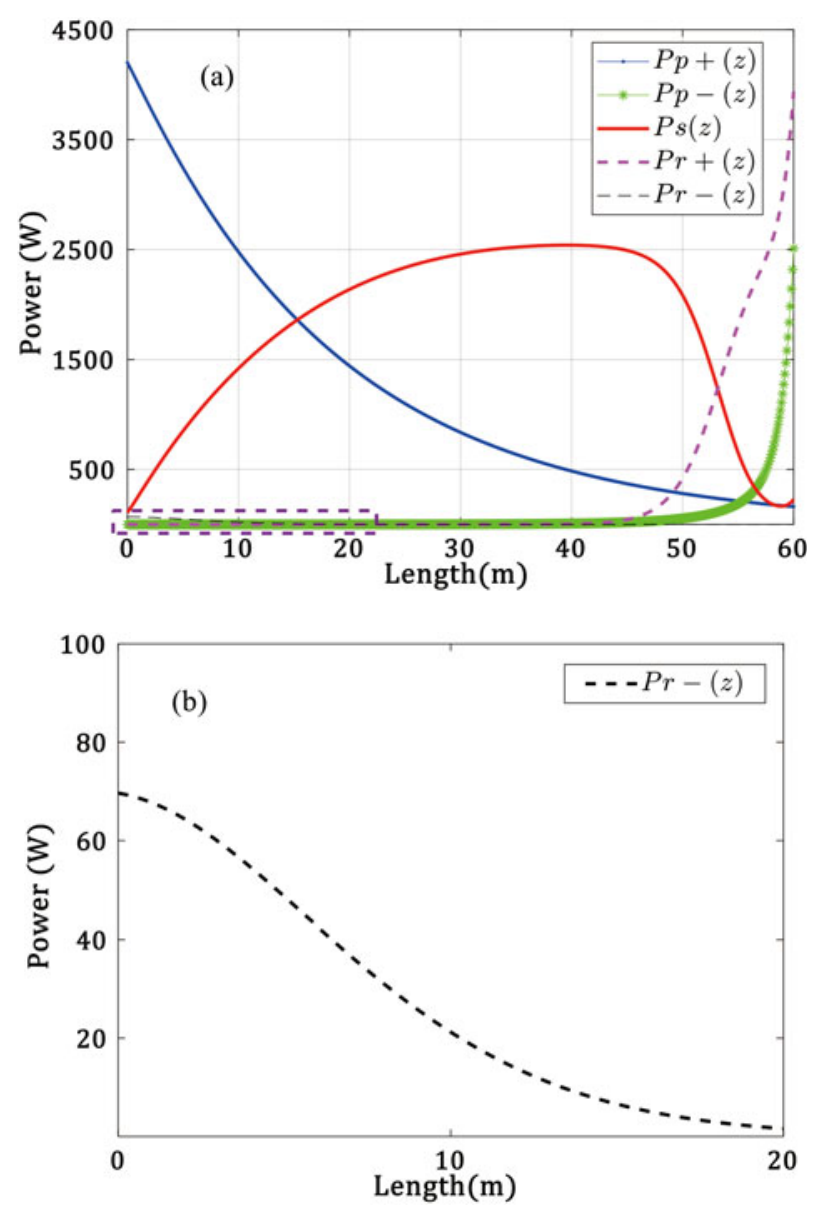

Figure 6. (a) Calculated power distributions of the (a) pump laser, signal laser and Raman laser along the fiber in the multi-frequency model $(L=$ $60 \mathrm{~m}$ ), and (b) backward Raman laser in the dotted box in (a).

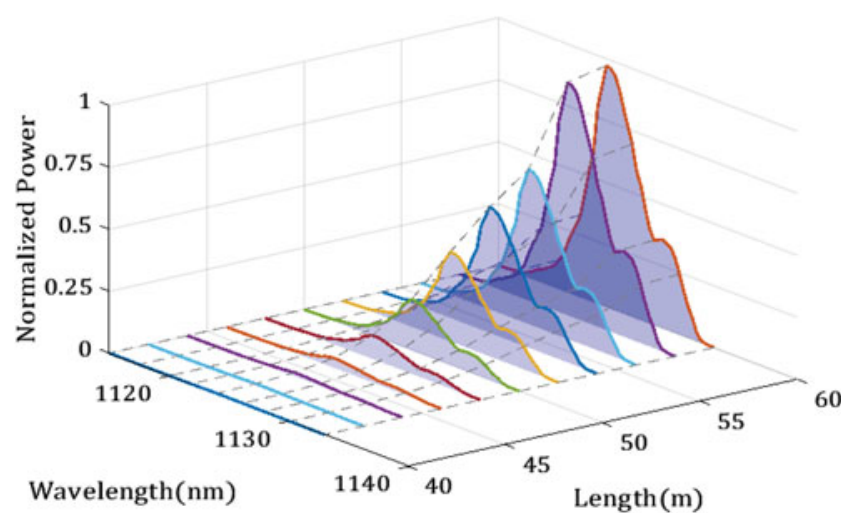

Figure 7. Transmission and amplification of Raman laser from 40 to $60 \mathrm{~m}$ (SRS has not been generated from 0 to $40 \mathrm{~m}$ ).

a flatter power distribution, the $1018 \mathrm{~nm}$ YDFLs pumping suffers from the less thermal effect than the $976 \mathrm{~nm}$ LDs pumping. Therefore, the temperature of gain fiber in $976 \mathrm{~nm}$ LDs pump configuration is higher ${ }^{[31-33]}$.
We analyzed the impact of different pump source on temperature distribution via simulation under the same pump power. The forward pump power and the backward pump power were set to $4200 \mathrm{~W}$ and $2510 \mathrm{~W}$, respectively. The result was shown in Figure 8. Two situations were analyzed to compare the difference. One of the situation has the same parameters with our actual experiment, while in the other situation only $976 \mathrm{~nm}$ LDs were used as pumping source. The simulation result showed that the highest temperature in the gain fiber of these two configurations was $36^{\circ}$ and $210^{\circ}$, respectively. In consideration of thermal effect, adopting $1018 \mathrm{~nm}$ YDFLs as pump source will have more potential for further power scaling.

\subsection{The analysis of the new peak at $1095 \mathrm{~nm}$}

As mentioned above, we found that a new peak at a wavelength of about $1095 \mathrm{~nm}$ appeared when the total output power exceeded $1360 \mathrm{~W}$, much smaller than the Raman threshold. Thus, we assumed that it was not related to the SRS. In addition, the wavelength of the new peak shifted from 1089 to $1095 \mathrm{~nm}$ while the total output power rose from 1360 to 4290 W, as shown in Figure 9.

Without the item of SRS, we integrated both sides of Equation (2) in the region $[0, L]$, obtaining the following expression of the gain coefficient $G$ :

$$
\begin{aligned}
G(\lambda)=\ln \frac{P_{s}(L)}{P_{s}(0)}= & \Gamma_{s}\left[\sigma_{a, s}(\lambda)+\sigma_{e, s}(\lambda)\right] \int_{0}^{L} N_{2}(z) \mathrm{d} z \\
& -\left[\sigma_{a, s}(\lambda) N+\alpha_{s}\right] L .
\end{aligned}
$$

It is clear that the gain coefficient $G$ was the function of wavelength. Figure 10 illustrated the exponential of gain coefficient when forward pumping power was $1800 \mathrm{~W}$. Owing to a very long length of gain fiber and high pump power, the maximum gain coefficient was located at $1095 \mathrm{~nm}$. At the beginning of the amplifier, the $1070 \mathrm{~nm}$ signal laser was dominant. However, the $1095 \mathrm{~nm}$ laser, only generated from ASE initially, was strongly amplified along the fiber because of much larger gain coefficient. Figure 11 presented the numerical simulation results of the power evolution along the fiber according to Equations (1)-(6). The gain coefficient $G$ increased in pace with gain fiber length growth. We found that the new wavelength of the laser only appeared when the gain fiber length was larger than $50 \mathrm{~m}$. The new peak can also be explained by resorption in a long YDF laser ${ }^{[34]}$.

\section{Experiment of the second-order Raman fiber laser}

To increase the Raman gain coefficient and produce a second- or higher-order Raman laser, we spliced another GDF after the gain fiber, as shown in Figure 12. The core and cladding diameters of the GDF were 25 and $400 \mu \mathrm{m}$, 


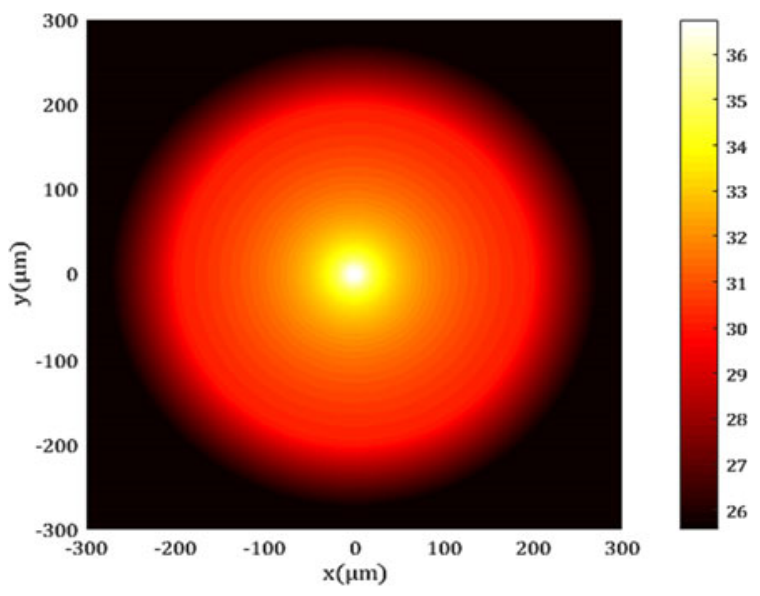

(a)

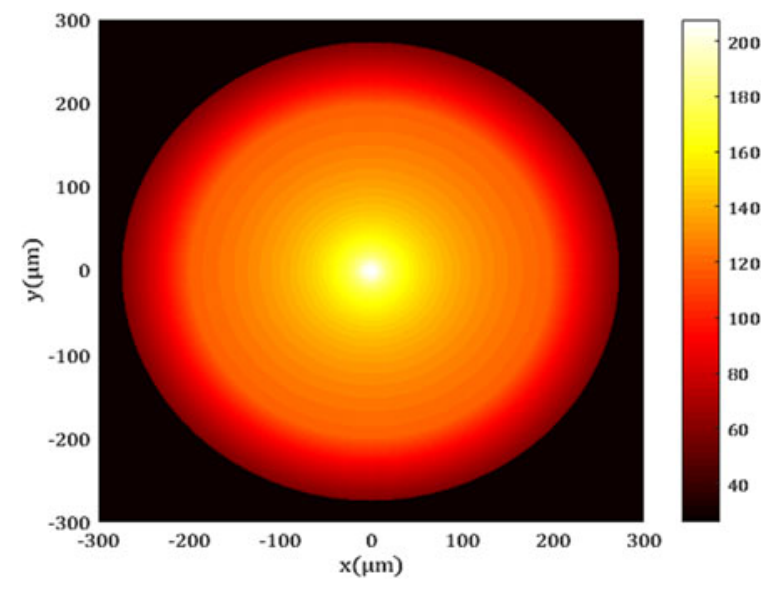

(b)

Figure 8. Temperature distribution at the input end of two situations. (a) The experiment; (b) only $976 \mathrm{~nm}$ LDs were used.

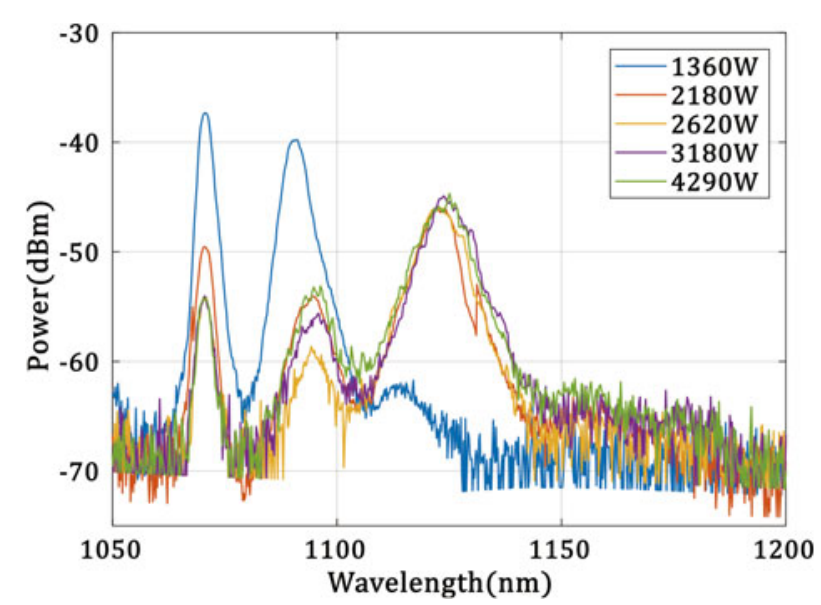

Figure 9. Output spectra under different powers (the length of YDF is $60 \mathrm{~m})$.

respectively, identical to the gain fiber. Figure 13 presented the output spectra when the length of the GDF was 50, 70, 80 and $100 \mathrm{~m}$.

Figure 13(a) indicated that the first-order Raman laser was still dominant when the GDF length is $50 \mathrm{~m}$. As the length of the GDF increases, the second-order Raman laser will replace the dominance of the first-order Raman laser. While the GDF was $70 \mathrm{~m}$, as shown in Figure 13(b), the center wavelengths of the first-, second- and thirdorder stimulated Raman lasers were 1122.5, 1182.7 and $1243.3 \mathrm{~nm}$, respectively. In addition, the output power of the second-order Raman laser was about $1500 \mathrm{~W}$, which accounts only for $45 \%$ of the total $3610 \mathrm{~W}$ output power. The $3 \mathrm{~dB}$ spectral width of the $1182.7 \mathrm{~nm}$ second-order Raman fiber was $14 \mathrm{~nm}$. However, the power of the second-order Raman laser was only approximately $5 \mathrm{~dB}$ higher than that of the first- or third-order Raman laser. For future work, to

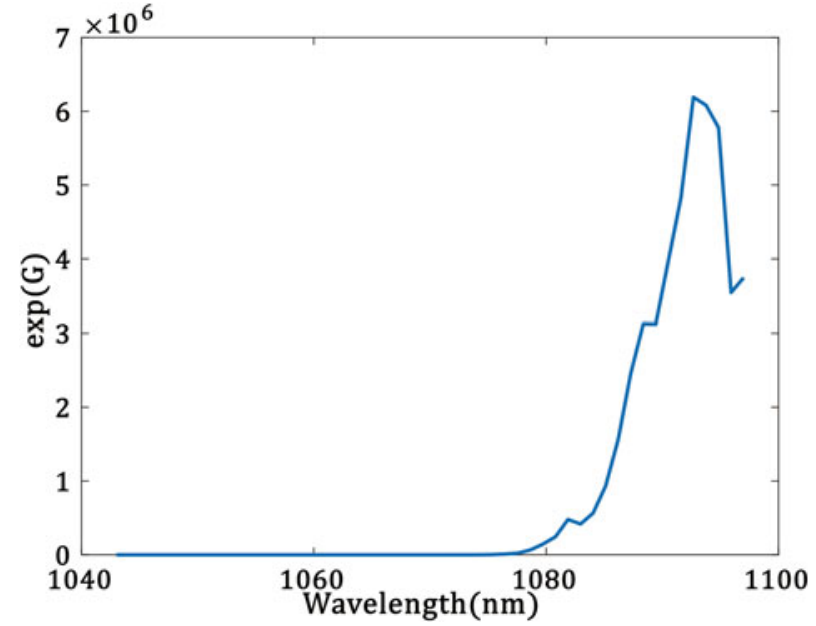

Figure 10. Exponential of gain coefficient versus wavelength (the length of YDF is $60 \mathrm{~m}$ ).

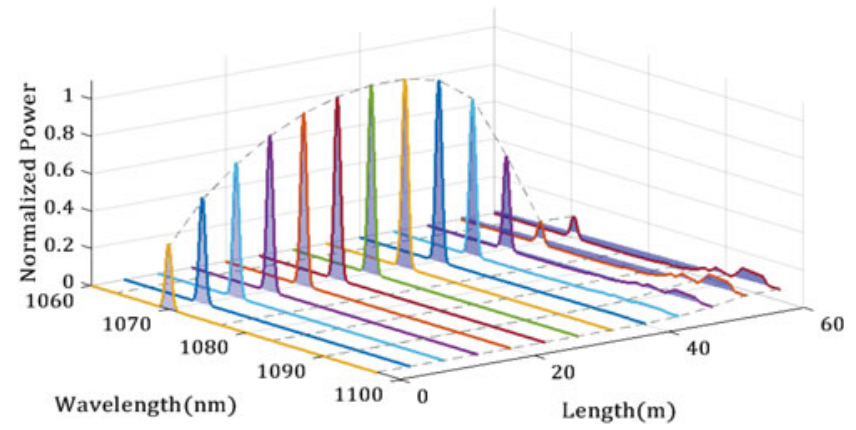

Figure 11. Generation of the new laser wavelength (the forward pump power is $1800 \mathrm{~W}$ ).

obtain a second-order Raman laser and suppress other-order Raman lasers, we are preparing to optimize the length of the YDF and GDF, and inject a seed comprising a 1070 and $1180 \mathrm{~nm}$ laser into the amplifier. 


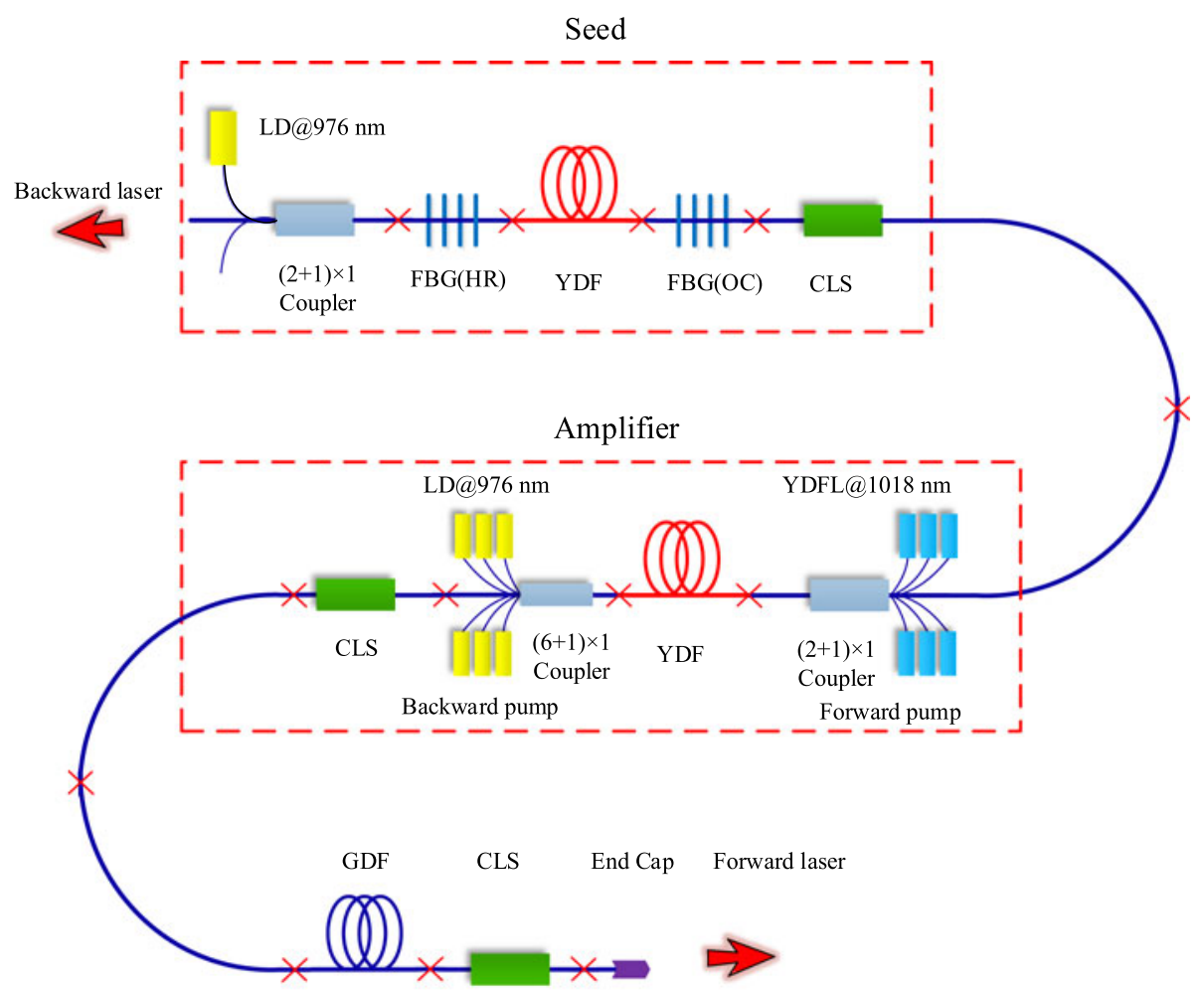

Figure 12. Experimental setup when the GDF is spliced after the YDF.
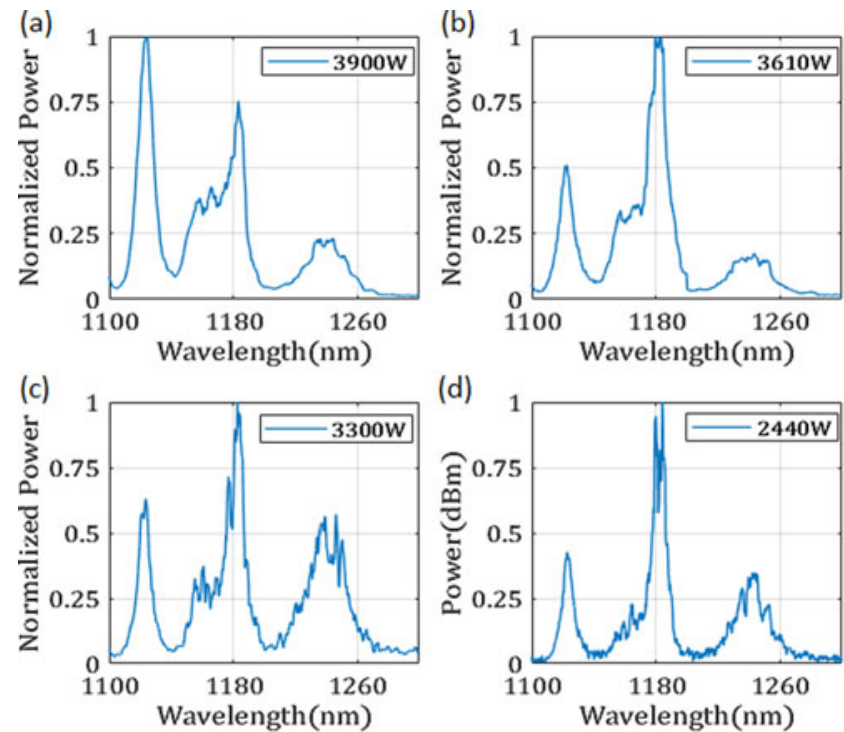

Figure 13. Output spectra and total power $(P)$ at different lengths (LG) of splicing GDF: LG, $P$ are (a) $50 \mathrm{~m}, 3900 \mathrm{~W}$, (b) $70 \mathrm{~m}, 3610 \mathrm{~W}$, (c) $80 \mathrm{~m}$, $3300 \mathrm{~W}$ and (d) $100 \mathrm{~m}, 2440 \mathrm{~W}$, respectively.

\section{Conclusions}

In this paper, we presented both the experimental realization and theoretical analysis of a $3.7 \mathrm{~kW}, 1123 \mathrm{~nm}$ Raman fiber laser system. The system adopted a dual-wavelength bidirectional pumping configuration, consisting of $1018 \mathrm{~nm}$ YDFLs and $976 \mathrm{~nm}$ LDs as the forward and backward pump sources, respectively. The usage of long-wavelength pump sources reduced the thermal effect caused by quantum defects. A 60-m-long, 25/400- $\mu \mathrm{m}$ YDF was employed as the Raman gain medium. The maximum output power was $3700 \mathrm{~W}$ while $4200 \mathrm{~W}$ of forward pump power and $2510 \mathrm{~W}$ of backward pump power were injected into the amplifier. The $3 \mathrm{~dB}$ spectral width of the $1123 \mathrm{~nm}$ Raman laser was $6.8 \mathrm{~nm}$. The backward Raman laser power was $65 \mathrm{~W}$ with a $3 \mathrm{~dB}$ spectral width of $5.2 \mathrm{~nm}$. Under a pump power of $3020 \mathrm{~W}$, the $M^{2}$ factor of the output laser was measured to be 2.18 . In addition, we proposed a multi-frequency model based on the steady-state rate equation. Taking the spectrum of the Raman gain and ASE into account, our model can resolve the spectra and power evolutions in both the forward and backward directions along the fiber. The numerical simulation results were in good agreement with the experimental results. The appearance of a new peak at about $1095 \mathrm{~nm}$ was also investigated theoretically and experimentally. Furthermore, a $1500 \mathrm{~W}, 1187 \mathrm{~nm}$ secondorder Raman laser was obtained by splicing another $70 \mathrm{~m}$ GDF after the YDF. The power scaling of the second-order Raman laser will be studied in future work. 


\section{Acknowledgements}

This work was supported in part by the National Natural Science Foundation of China (Nos. 61675114 and 61875103) and the Tsinghua University Initiative Scientific Research Program (No. 20151080709).

\section{References}

1. L. R. Taylor, Y. Feng, and D. B. Calia, Opt. Express 18, 8540 (2010).

2. Y. Feng, L. Zhang, and H. Jiang, Proc. SPIE 9344, 93440U (2015).

3. G. Qin, S. Huang, Y. Feng, A. Shirakawa, and K. Ueda, Opt. Lett. 30, 269 (2005).

4. X. Du, H. Zhang, H. Xiao, P. Ma, X. Wang, P. Zhou, and Z. Liu, Ann. Phys.-Berlin 528, 649 (2016).

5. X. Wang, P. Zhou, Y. Miao, H. Zhang, H. Xiao, X. Wang, and Z. Liu, J. Opt. Soc. Am. B 31, 2476 (2014).

6. H. Zhang, P. Zhou, X. Wang, X. Du, H. Xiao, and X. Xu, Opt. Express 23, 17138 (2015).

7. S. D. Jackson, Nat. Photon. 6, 423 (2012).

8. F. Beier, C. Hupel, S. Kuhn, S. Hein, J. Nold, F. Proske, B. Sattler, A. Liem, C. Jauregui, J. Limpert, N. Haarlammert, T. Schreiber, R. Eberhardt, and A. Tünnermann, Opt. Express 25, 14892 (2017).

9. H. Zhang, H. Xiao, P. Zhou, K. Zhang, X. Wang, and X. Xu, Appl. Phys. Express 7, 52701 (2014).

10. H. Zhang, H. Xiao, P. Zhou, X. Wang, and X. Xu, Opt. Express 22, 10248 (2014)

11. H. Zhang, R. Tao, P. Zhou, X. Wang, and X. Xu, IEEE Photon. Technol. Lett. 27, 628 (2015).

12. L. Zhang, C. Liu, H. Jiang, Y. Qi, B. He, J. Zhou, X. Gu, and Y. Feng, Opt. Express 22, 18483 (2014).

13. P. Ma, H. Zhang, L. Huang, X. Wang, P. Zhou, and Z. Liu, Opt. Express 23, 26499 (2015).

14. H. Ying, J. Cao, Y. Yu, M. Wang, Z. Wang, and J. Chen, Optik 144, 163 (2017).
15. Q. Xiao, P. Yan, D. Li, J. Sun, X. Wang, Y. Huang, and M. Gong, Opt. Express 24, 6758 (2016).

16. Y. Glick, Y. Shamir, M. Aviel, Y. Sintov, S. Goldring, N. Shafir, and S. Pearl, Opt. Lett. 43, 4755 (2018).

17. Y. Chen, J. Leng, H. Xiao, T. Yao, and P. Zhou, in 8 th EPSQEOD Europhoton Conference (Barcelona, Spain, 2018), paper TuM1.6.

18. Y. Chen, H. Xiao, J. Xu, J. Leng, and P. Zhou, Appl. Opt. 55, 3824 (2016).

19. M. Rini, I. Cristiani, and V. Degiorgio, IEEE J. Quantum Electron. 36, 1117 (2000).

20. P. Yan, X. Wang, D. Li, Y. Huang, J. Sun, Q. Xiao, and M. Gong, Opt. Lett. 42, 1193 (2017).

21. P. Yan, X. Wang, Z. Wang, Y. Huang, D. Li, Q. Xiao, and M. L. Gong, IEEE J. Sel. Top. Quantum Electron. 24, 0902506 (2018).

22. Q. Xiao, P. Yan, H. Ren, X. Chen, and M. Gong, J. Lightwave Technol. 31, 2715 (2013).

23. Q. Xiao, H. Ren, P. Yan, X. Chen, and M. Gong, Opt. Commun. 300, 220 (2013).

24. Y. Feng, Raman Fiber Lasers (Springer, New York, 2017).

25. M. Chen, A. Shirakawa, X. Fan, K. Ueda, C. B. Olausson, J. K. Lyngsø, and J. Broeng, Opt. Express 20, 21044 (2012).

26. R. Paschotta, J. Nilsson, A. C. Tropper, and D. C. Hanna, IEEE J. Quantum Electron. 33, 1049 (1997).

27. A. Hardy and R. Oron, IEEE J. Quantum Electron. 33, 307 (1997).

28. R. H. Stolen, C. Lee, and R. K. Jain, J. Opt. Soc. Am. B 1, 652 (1984).

29. D. Hollenbeck and C. D. Cantrell, J. Opt. Soc. Am. B 19, 2886 (2002).

30. Y. Glick, V. Fromzel, J. Zhang, A. Dahan, N. Tergabrielyan, R. K. Pattnaik, and M. Dubinskii, Laser Phys. Lett. 13, 65101 (2016).

31. C. Jauregui, J. Limpert, and A. Tünnermann, Nat. Photon. 7, 861 (2013).

32. T. Yao, J. Ji, and J. Nilsson, J. Lightwave Technol. 32, 429 (2014).

33. P. Zhou, H. Xiao, J. Leng, J. Xu, Z. Chen, H. Zhang, and Z. Liu, J. Opt. Soc. Am. B 34, A29 (2017).

34. J. Nilsson, W. A. Clarkson, R. Selvas, J. K. Sahu, P. W. Turner, S. Alam, and A. B. Grudinin, Opt. Fiber Technol. 10, 5 (2004). 